\title{
ITR-Seq, a next-generation sequencing assay, identifies genome-wide DNA editing sites in vivo following adeno-associated viral vector-mediated genome editing
}

\author{
Camilo Breton ${ }^{\dagger}$, Peter M. Clark, Lili Wang, Jenny A. Greig and James M. Wilson º
}

\begin{abstract}
Background: Identifying nuclease-induced double-stranded breaks in DNA on a genome-wide scale is critical for assessing the safety and efficacy of genome editing therapies. We previously demonstrated that after administering adeno-associated viral (AAV) vector-mediated genome-editing strategies in vivo, vector sequences integrated into the host organism's genomic DNA at double-stranded breaks. Thus, identifying the genomic location of inserted AAV sequences would enable us to identify DSB events, mainly derived from the nuclease on- and off-target activity.

Results: Here, we developed a next-generation sequencing assay that detects insertions of specific AAV vector sequences called inverted terminal repeats (ITRs). This assay, ITR-Seq, enables us to identify off-target nuclease activity in vivo. Using ITR-Seq, we analyzed liver DNA samples of rhesus macaques treated with AAV vectors expressing a meganuclease. We found dose-dependent off-target activity and reductions in off-target events induced by further meganuclease development. In mice, we identified the genomic locations of ITR integration after treatment with Cas9 nucleases and their corresponding single-guide RNAs.

Conclusions: In sum, ITR-Seq is a powerful method for identifying off-target sequences induced by AAV vectordelivered genome-editing nucleases. ITR-Seq will help us understand the specificity and efficacy of different genomeediting nucleases in animal models and clinical studies. This information can help enhance the safety profile of geneediting therapies.
\end{abstract}

Keywords: Genome editing, Off-targets, Next-generation sequencing, In vivo, Editing, AAV integration

\section{Background}

Genome-editing therapies disrupt normal gene function by creating double-stranded breaks (DSBs) in DNA, induced by a nuclease such as CRISPR-Cas9, within a targeted locus. The host cell subsequently repairs these DSBs, thus resulting in edited alleles that can contain insertions and deletions (indels) or more complex genomic rearrangements

\footnotetext{
* Correspondence: wilsonjm@upenn.edu

${ }^{\dagger}$ Camilo Breton and Peter M. Clark contributed equally to this work. Gene Therapy Program, University of Pennsylvania Perelman School of Medicine, 125 South 31st Street, Suite 1200, Philadelphia, PA 19104, USA
}

[1]. The specificity of these nucleases is conferred by either 1) a single-guide RNA (sgRNA) in the case of the CRISPRCas system [2]; 2) a rational design and evolution of sequence-specific DNA-binding domains fused to a nuclease (e.g., TALENs or zinc fingers [3]); or 3) engineered versions of restriction enzymes (e.g., meganucleases) $[4,5]$. In spite of efforts by the scientific community to increase the on-target efficiency and specificity of these nucleases, they often exhibit off-target editing effects. Assessing the location and frequency of nuclease target sites is a critical step

C C The Author(s). 2020 Open Access This article is licensed under a Creative Commons Attribution 4.0 International License, which permits use, sharing, adaptation, distribution and reproduction in any medium or format, as long as you give appropriate credit to the original author(s) and the source, provide a link to the Creative Commons licence, and indicate if changes were made. The images or other third party material in this article are included in the article's Creative Commons licence, unless indicated otherwise in a credit line to the material. If material is not included in the article's Creative Commons licence and your intended use is not permitted by statutory regulation or exceeds the permitted use, you will need to obtain permission directly from the copyright holder. To view a copy of this licence, visit http://creativecommons.org/licenses/by/4.0/. The Creative Commons Public Domain Dedication waiver (http://creativecommons.org/publicdomain/zero/1.0/) applies to the data made available in this article, unless otherwise stated in a credit line to the data. 
towards evaluating the safety and efficacy of genomeediting therapies.

Researchers have developed a variety of approaches to identify and quantify the on- and off-target activity of genome editing nucleases to better understand the elements that govern nuclease specificity and to improve the safety profile of these therapies [6]. Nuclease specificity and activity can be studied by first identifying the DSB, either in cultured cells or in animal models, created as a consequence of their nuclease activity $[7,8]$. Approaches for determining nuclease specificity include cell-free methods, such as SiteSeq [9], Digenome-seq [10], and Circle-Seq [11], and in vitro methods, such as GUIDE-Seq [12] and IntegrativeDeficient Lentiviral Vectors Capture [13, 14]. In addition, during the preparation of this manuscript, Hanlon et al. published an assay, similar to ours, to identify AAV integration after CRISPR-Cas9-mediated editing to identify onand off-target sites [15].

One of the currently preferred methods for characterizing the off-target activity of nucleases is GUIDE-Seq because 1) it requires a minimal number of components; 2 ) software is readily available to identify off-target sites; and 3 ) it can detect low-abundance off-target sites. However, these in vitro analyses might not accurately predict the number and rate of off-target activity in vivo, as the differences in nuclease intracellular levels and the chromatin state between these two conditions (in vitro and in vivo) might affect the editing activity within the on- and off-target sites [16].

Assays such as Breaks Labeling In Situ and Sequencing (BLISS) [17] or End-Seq [18] require an initial cell-fixation step to preserve the free DSB ends. The BLISS approach captures these ends by ligating adapters containing a T7 promoter. An in vitro transcription assay then amplifies the adjacent DNA, producing RNA to construct Illumina libraries for next-generation sequencing (NGS) analysis [17]. The End-Seq method captures DSB ends by ligating biotinylated hairpin adapters [18]. NGS analysis of BLISS and End-Seq NGS libraries makes it possible to accurately determine the sites of nuclease-induced DSBs by selecting sites with homology to the intended target sequence. However, this analysis is only capable of identifying DSBs present at a discreet time point, given that the adapters can only be ligated to free DSB ends. Therefore, these assays cannot capture repaired DSBs created at earlier time points.

To accelerate the translation of gene-editing platform technologies into the clinic, we need to accurately quantify the location and frequency of nuclease-induced DSBs in vivo. Our current strategy to edit endogenous genes within the liver involves intravenous (IV) administration of a gene therapy vector expressing a nuclease. By using adeno-associated virus (AAV) serotype 8 (AAV8), we can effectively target hepatocytes and induce nucleasemediated genome editing. However, due to the creation of DSBs following genome editing, there is the potential of integrating a fragment of the vector sequences such as inverted terminal repeat (ITR) sequences [19-27]. Therefore, determining the genomic location of inserted ITR sequences would enable us to characterize on- and off-target DSB events.

Here, we developed an assay called ITR-Seq to determine the number and genomic location of these integration events in vivo. We designed an ITR sequence-specific primer to amplify the ITR-genomic DNA junction that occurs after the integration of ITRs directly from primary tissue. We used ITR-Seq to identify and rank genomewide target sites for five independent nucleases in vivo. Our results indicate that ITR-Seq is a highly sensitive and specific assay for in vivo sites of DSBs and significantly advances our ability to assess the safety and efficacy of translational genome-editing therapies.

\section{Results}

Developing the ITR-Seq assay to evaluate meganuclease activity in non-human primates

We sought to develop a methodology for unbiased, genome-wide identification of sites of ITR integration. Using the AAV ITR as a tag for identifying DSBs, we can measure the off-target activity of genome-editing nucleases in vivo. Our method is based on previous research that demonstrated AAV ITR sequence integration into the host's genomic DNA after the occurrence of DSBs [21-25, 27-31].

We reanalyzed the NGS reads that were generated in a previous study where we characterized the on-target regions following administration of AAV vectors expressing two generations of a meganuclease (AAV8-M1PCSK9 and AAV8-M2PCSK9) [26]. Our aim was to identify the most common AAV ITR sequences that were integrated into the meganuclease on-target loci in the PCSK9 gene. Based on a peak in absolute frequency at position 82 of the AAV2 reference genome (Fig. 1a), we determined that the most frequent base position of ITR integration occurs $5^{\prime}$ upstream of the Rep-binding element. We used this information to design an ITR-specific primer that hybridizes 5' upstream of the observed ITR-integration start site (shown in red in Fig. 1b). We used this primer in a novel NGS assay, based on anchored multiplexed PCR, to identify the ITR-genomic DNA junction following insertional mutagenesis (Fig. 1c). We named this method ITR-Seq.

To use the ITR-Seq assay for sample analysis, we first isolated DNA from the tissues of animals treated with nuclease-expressing AAV vectors. We sheared the DNA and ligated it to Y-adapters, as described in previous reports [12]. Following two rounds of PCR using the ITRspecific primer described above and adapter-specific primers, we produced NGS-compatible libraries. After sequencing, we computationally identified the resulting amplicons that contain both the amplified ITR sequence 


\section{a}

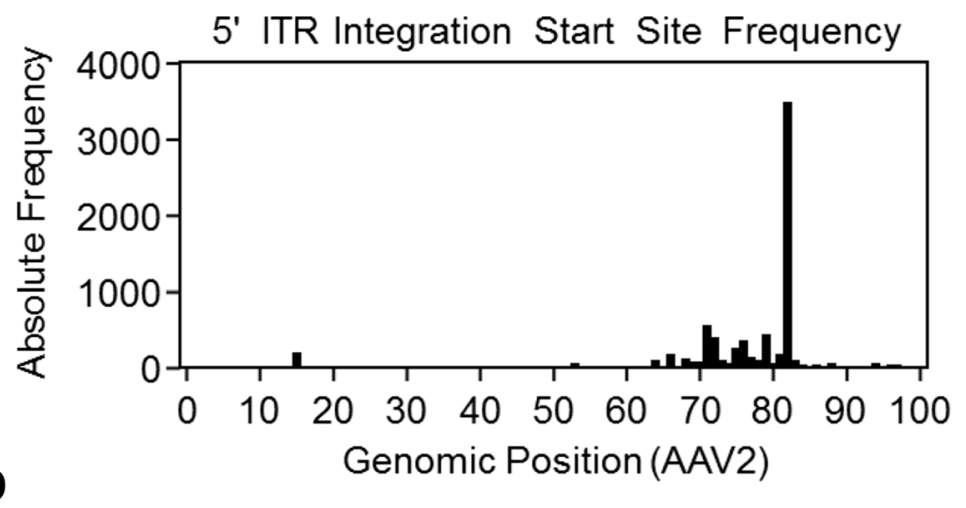

b Genomic Position (AAV2)

$\mathrm{RBE} \mathrm{E}^{\prime}$

$\mathrm{B}^{\prime}$ $B$ Most Frequent Start Site

A $3^{3}$

GCCTCAGTGAGCGAGCGAGCGCCAGAGAGGGAGTGGCCAACTCCATCACTAGGGTTCCTTGT

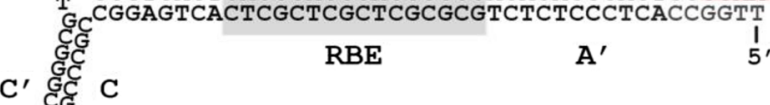

$\mathrm{C}^{\prime}$

$C$ 1

Nuclease-induced DSB

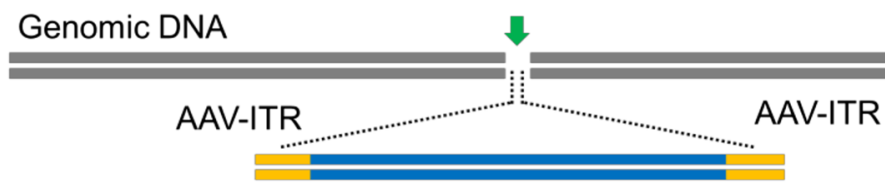

Partial or full-length rAAV integration in DSB site $\downarrow$
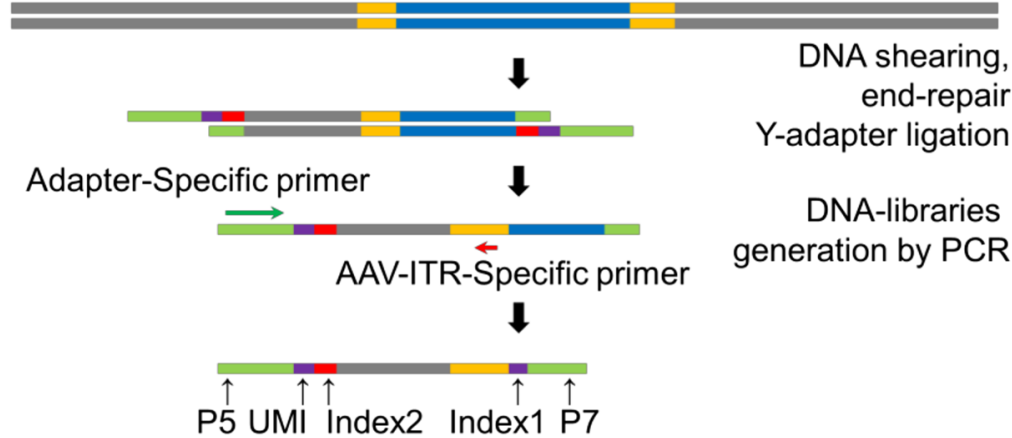

\section{$\downarrow$}

Next-generation sequencing and analysis

Fig. 1 Sequence analysis of AAV ITRs integrated into genomic DNA. a. Meta-analysis of on-target AMP-Seq data for all AAV8-M1PCSK9- and AAV8-M2PCSK9-treated liver samples (SRR6343442). Our goal was to identify the most frequent ITR integration start site within the vector ITR. $\mathbf{b}$. Secondary structure of the AAV2 5' ITR (NC_001401.2). The most frequently integrated start site position is shown. The ITR-Seq primer (GSP_ITR3.AAV2) binding site is highlighted in red. A-A', B-B', and C-C', palindromic arms; RBE, Rep-binding element; TRS, terminal resolution site. c. Schematic diagram of the ITR-Seq protocol used for genome-wide identification of ITR integration sites 
and adjacent genomic DNA sequences. We also determined the location and frequency of genome-wide ITR integration sites. By requiring that the ITR integrates in both the forward and reverse strand orientations, we aimed to further reduce the number of false positives and identify high-confidence ITR-integration sites. For each sample, we produced a rank-ordered list of nuclease target sites (ITR-Seq rank) and sorted the sites in decreasing order by the total number of observed ITRintegration events per locus (ITR-Seq reads). We generated ITR-Seq reports at the end of the computational analysis (Dataset S1 and S2) with the most probable offtarget sequence (based on homology to the intended target sequence), the genomic location, and the ITR-Seq rank (according to the number of NGS reads mapping to the corresponding locus).

\section{ITR-Seq identifies nuclease off-target sites in rhesus macaques}

We next used the ITR-Seq assay to further analyze the onand off-target effects of meganucleases in rhesus macaques that had previously been administered AAV8-M1PCSK9 or AAV8-M2PCSK9 (Fig. 2). As previously described, rhesus macaques received either one of three doses of AAV8M1PCSK9 $\left[3 \times 10^{13}\right.$ genome copies $(\mathrm{GC}) / \mathrm{kg}, 6 \times 10^{12} \mathrm{GC} /$ $\mathrm{kg}$, or $\left.2 \times 10^{12} \mathrm{GC} / \mathrm{kg}\right]$ or a single dose of AAV8-M2PCSK9 $\left(6 \times 10^{12} \mathrm{GC} / \mathrm{kg}\right)$ [26]. We took a liver biopsy of each macaque on days 17 and 128 post-vector administration to evaluate on- and off-target editing. Across all samples from meganuclease-treated macaques, the top ITR-Seq rank was the on-target locus (PCSK9 target site sequence TGGACC TCTTTGCCCCAGGGGA, chr1:54708864-54,708,885) [26]. For both generations of the meganuclease (i.e., AAV8M1PCSK9 and AAV8-M2PCSK9), the number off-target sites identified by ITR-Seq depended on both the administered vector dose and the sample time point. The number of off-target sites decreased as a function of time (e.g., day 17 had more off-target sites than day 128; see Fig. 2a). We observed a dose-dependent effect among the non-human primates treated with AAV-M1PCSK9, where the highest AAV-M1PCSK 9 dose $\left(3 \times 10^{13} \mathrm{GC} / \mathrm{kg}\right)$ resulted in the highest number of off-target sites (2332 off-target sites at d17) while those administered with the lowest tested dose $\left(2 \times 10^{12} \mathrm{GC} / \mathrm{kg}\right)$ resulted in the lowest number of offtarget sites for this group (120 and 138 off-target sites at d17, Fig. 2a). Animals that received the second-generation engineered meganuclease M2PCSK9 had fewer identified off-target sites than animals treated with the firstgeneration meganuclease M1PCSK9 at the same dose. In total, we observed 1170 different off-target sites after administering AAV8-M1PCSK9 at a dose of $6 \times 10^{12} \mathrm{GC} / \mathrm{kg}$. By contrast we only observed 194 and 105 off-target sites in the two macaques that received AAV8-M2PCSK9 at the same dose (Fig. 2a).
We then assessed the frequency of AAV integration in the on- and off-target sites by dividing the total number of ITR-Seq reads in the on- and off-target regions by the total number of ITR-Seq reads (on- and off-target). The distribution of ITR-Seq reads for each treated non-human primate at day 17 and d128 is shown in Figure S1. Interestingly, we observed an increase in the on-target \% from $\mathrm{d} 17$ to $\mathrm{d} 128$, indicating that by day 128 between 60 and 90\% of the AAV-integrated sequences are in the on-target region (Figure S1). This AAV integration frequency was significantly different (Wilcoxon singed-rank test) between the on- and off-target regions $(p=0.03125)$ at day 128 post AAV administration.

We randomly selected a subset of ITR-Seq-identified offtarget sites from the results of day 17 liver samples from macaques treated with $3 \times 10^{13}$ or $6 \times 10^{12} \mathrm{GC} / \mathrm{kg}$ of AAV8-M1PCSK9. We then investigated the presence of ITR sequences in these loci by AMP-Seq, as previously described [26, 32], using gene-specific primers flanking the identified off-target sequences (Additional File 1: Table S1). We found reads containing ITR sequences in 24 (for the $3 \times 10^{13} \mathrm{GC} / \mathrm{kg}$ dose) or 21 (for the $6 \times 10^{12} \mathrm{GC} / \mathrm{kg}$ dose) out of 27 interrogated loci, with the highest percentage of ITR integration (ITR-containing reads) corresponding to those off-target sites with high ITR-Seq rank (Additional File 1: Table S1). Importantly, in both animals we found the highest level of editing in those loci with the highest ITRSeq rank, suggesting a correlation between the ITR-Seq rank, the percentage of ITR integration, and the indel percentage. For some of these loci, we could not detect ITR sequences integrated by the AMP-Seq assay (Additional File 1: Table S1); this could be due to a lower sensitivity of the AMP-Seq assay to detect ITR integration, as the ITR-Seq method uses the actual ITR sequences as a starting point for the amplification. Similarly, we were able to observe ITR sequences in a couple of sites not shown in the ITRSeq results (e.g., 20:359062-359,285 and 7:165269225-165, 269,449 for liver samples of animals treated with $3 \times 10^{13}$ GC/kg of AAV8-M1PCSK9; Dataset S1). However, we found these sites with the ITR-Seq method in animals treated with $6 \times 10^{12} \mathrm{GC} / \mathrm{kg}$ of AAV8-M1PCSK9 (Dataset S1), suggesting that our current protocol does not capture $100 \%$ of the ITR integration sites. Therefore, the sensitivity of the ITR-Seq method can be improved.

We then annotated the identified sites of ITR integration based on the function of the DNA region (Fig. 2b). Regardless of the number of identified nuclease target sites, the genomic distribution of target sites (intergenic, intronic, or exonic regions) was reproducible among meganuclease-administered macaques. Generally, most target sites reside within introns, followed by intergenic regions of the genome (Fig. $2 \mathrm{~b}$ ). The meganucleases we evaluated here have a 22-nucleotide target site within the PCSK9 gene. Therefore, we evaluated the number of 


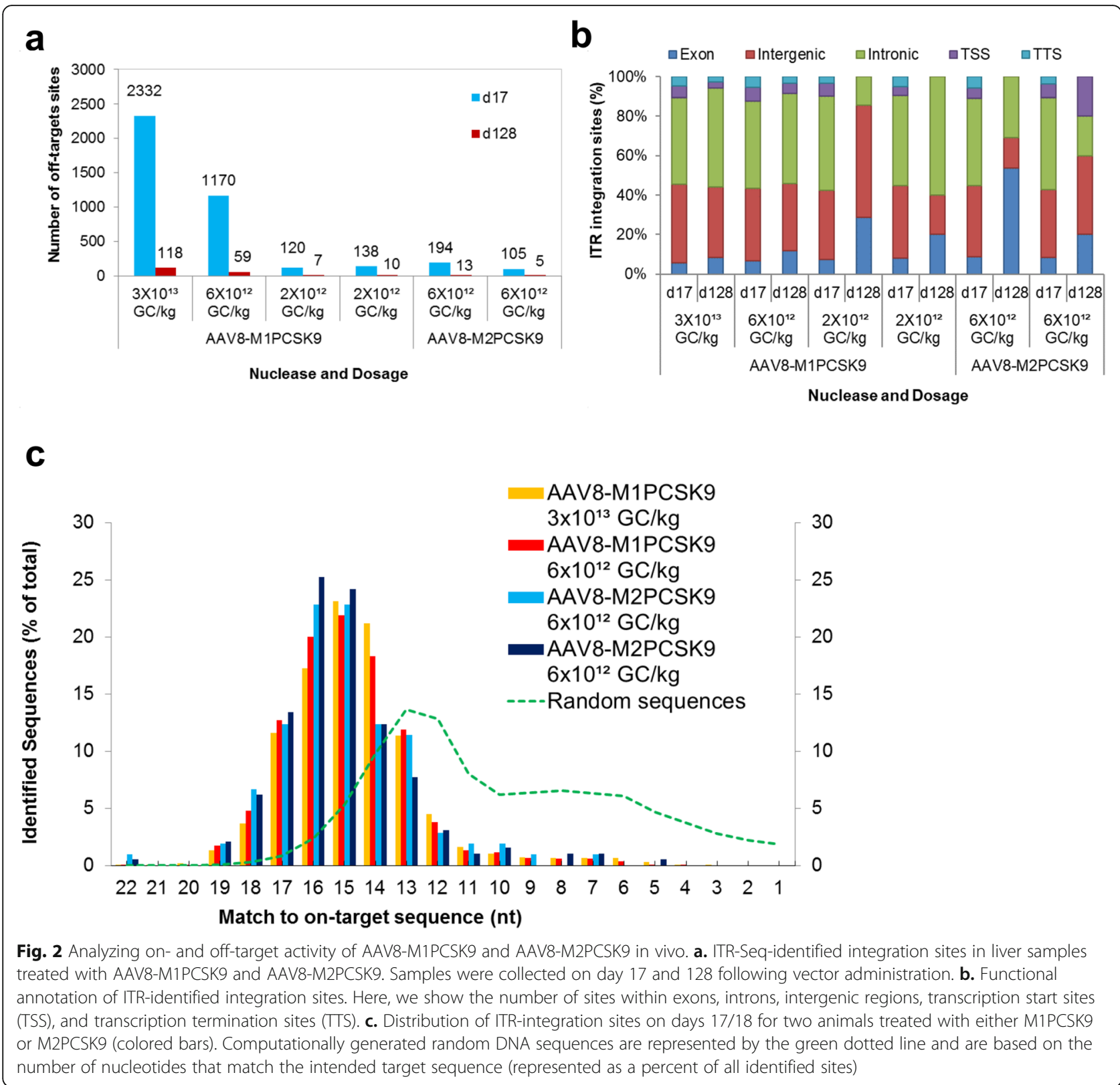

conserved nucleotides between the target DNA sequence and the DNA sequence of each off-target site (Fig. 2c). The distribution of matches to the on-target sequence appeared to follow a Gaussian distribution with a mean of 15-16 nucleotides. This indicates that the majority of ITR-Seq-identified off-target sites have six or seven mismatches between the targeted DNA sequence motif and the genomic DNA sequence of each target site (Fig. 2c). To assess if this level of homology resulted from editing in sequences similar to the meganuclease target sequence or simply due to chance, we generated ten million random DNA regions (40 bp in length) within the rhesus macaque genome. We then attempted to identify the sequence that was most similar to the meganuclease target sites using the same algorithm from the ITR-Seq protocol (Fig. 2c, green line). Unlike ITR-Seq-identified sequences, random sequences share an average of 11-12 mismatches with the intended target sequence. This indicates that the ITR is mostly integrated in the targets with a certain degree of homology to the intended target sequence. We then analyzed a subset of these off-target sequences to evaluate the distribution of mismatches between the on-target sites and a subset of the top-ranked off-target sites. We found that these mismatches were more likely to occur in particular nucleotide positions (nucleotides 1, 4, 12, 13, 14, and 21; Figure S2), while some nucleotides remained unchanged between the on- and off-target sites (nucleotides $2,3,5,11,15,16$, and 18; Figure S2). 
Comparing GUIDE-Seq and ITR-Seq identification of offtarget sites

For each meganuclease (M1PCSK9 and M2PCSK9), we had previously performed GUIDE-Seq analyses on LLC-MK2 cells transfected with plasmids to express the nucleases in order to identify off-target sites in vitro [26]. We selected the M1PCSK9 and M2PCSK9 off-target locations from the list of GUIDE-Seq-identified off-target sites in vitro. Using rhesus macaques, we then validated these predicted offtarget sites in vivo using amplicon sequencing of off-target loci [26]. Both amplicon sequencing [26] and ITR-Seq (Fig. 2a) exhibited a dose- and time-dependent reduction in offtarget editing efficiency. We compared these previous results with our evaluation of off-target sites in vivo using ITR-Seq (Fig. 3). By identifying off-target sites with no homology to the indented target sequence, we found approximately the same number of off-target sites across two independent experiments using either M1PCSK9 (1093 and 1499, for GUIDE-Seq experiment 1 and 2, respectively) or M2PCSK9 (568 and 651, for GUIDE-Seq experiment 1 and 2 , respectively). We compared sites that were identified by both GUIDE-Seq in vitro experiments to the off-target sites identified by ITR-Seq. For this study in rhesus macaques, we performed ITR-Seq on DNA samples from liver biopsies taken on day 17 post-nuclease administration. We compared the GUIDE-Seq- and ITR-Seq-identified off-target sites in macaques that received a dose of $3 \times 10^{13} \mathrm{GC} / \mathrm{kg}$ of AAV8-M1PCSK9 (Fig. 3a), $6 \times 10^{12} \mathrm{GC} / \mathrm{kg}$ of AAV8M1PCSK9 (Fig. 3b), or $6 \times 10^{12} \mathrm{GC} / \mathrm{kg}$ of AAV8-M2PCSK9 (two animals; Fig. 3c, d). Most (71.9-82.9\%) off-target sites were identified exclusively by ITR-Seq, and not by GUIDESeq (see colored sections of Fig. 3). Interestingly, among animals that received the second-generation nuclease (M2PCSK9), both ITR-Seq and GUIDE-Seq identified fewer off-target sites (see white sections of Fig. 3).

We have validated a set of GUIDE-Seq-identified highand low-rank off-target sites, which were ranked according to the number of reads [26]. We did this by quantifying the indel percentage, which we determined using amplicon sequencing of off-target loci in samples taken from macaques administered with meganucleases on days 17/18 and 128/129 [26]. We counted off-target sites

\section{a}

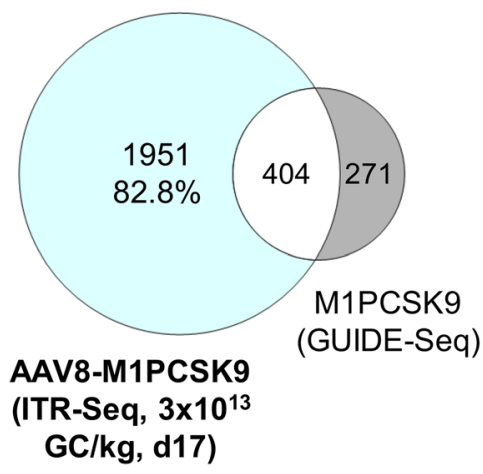

C

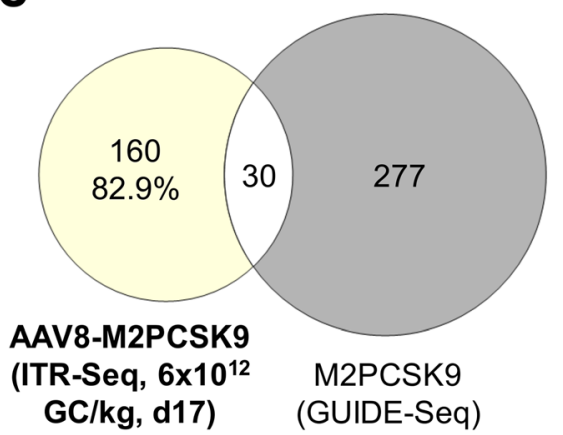

b

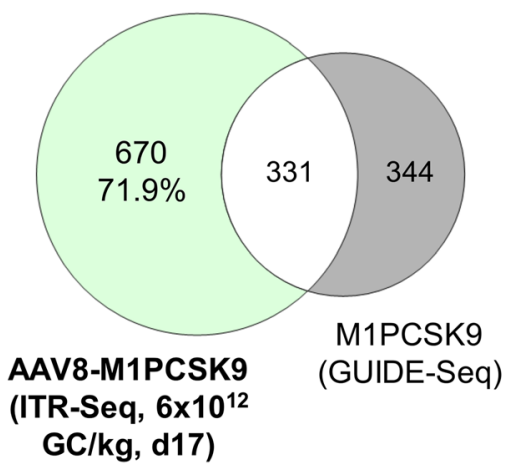

d

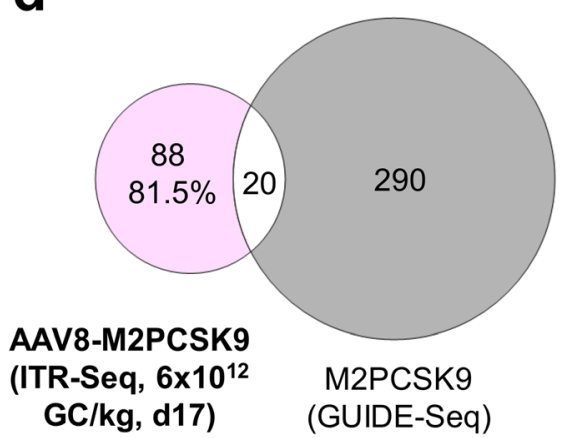

Fig. 3 Comparing GUIDE-Seq and ITR-Seq in terms of off-target identification. Sample set intersections of identified target sites obtained from in vivo ITR-Seq (coloured) from two doses of AAV8-M1PCSK9 $\left(3 \times 10^{13} \mathrm{GC} / \mathrm{kg}\right.$; panel $\mathbf{a}$; and $6 \times 10^{12} \mathrm{GC} / \mathrm{kg}$; panel b) or one dose of AAV8M2PCSK9 $\left(6 \times 10^{12} \mathrm{GC} / \mathrm{kg}\right.$; panels $\mathbf{c}$ and $\left.\mathbf{d}\right)$. We obtained target sites on day 17 post-AAV administration. In vitro GUIDE-Seq for M1PCSK9 or M2PCSK9 is shown in gray. Off-target sites identified by ITR-Seq but not by GUIDE-Seq (coloured sections) are indicated as a percent of the total number of off-target sites that were identified by in vivo ITR-Seq. White sections of the Venn diagrams show the proportion of off-target sites that were identified by both ITR-Seq and GUIDE-Seq 
that exhibited a significantly higher indel percentage than untreated peripheral blood mononuclear cell (PBMC) DNA control samples as positive (bold typeface in Additional File 1: Table S2).

Here, we evaluated whether ITR-Seq can identify positive GUIDE-Seq off-target sites. Given that we re-analyzed the same DNA used to validate the GUIDE-Seq off-target sites, we were able to assess the sensitivity of our method in detecting the positive off-target loci. Among macaques administered with $3 \times 10^{13}$ and $6 \times 10^{12} \mathrm{GC} / \mathrm{kg}$ of AAV8M1PCSK9, ITR-Seq only failed to identify two high-rank and two low-rank positive GUIDE-Seq off-target sites (S3 Table). Among macaques administered with AAV8M2PCSK9, ITR-Seq correctly identified most of the positive off-target sites and only missed three low-rank positive off-target sites in one animal and three high-rank positive off-target sites in the other macaque. Taken together, these results clearly indicate that ITR-Seq and not GUIDE-Seq could identify the vast majority of in vivo off-target sites. This suggests that unlike amplicon sequencing of GUIDESeq-predicted off-target sites, ITR-Seq more accurately examines the activity of AAV-delivered nucleases in vivo.

\section{Evaluating ITR-Seq assay as a tool for identifying guide RNA-dependent nuclease off-target sites in mice}

We tested whether ITR-Seq can detect the on- and offtarget activity of a variety of guide RNA-dependent nucleases (i.e., SaCas9, LbCpf1, and AsCpf1) that are commonly used in preclinical studies. We also evaluated whether ITR-Seq analysis is compatible with the distinct types of DSB ends created by these nucleases (blunt for SaCas9 and 5' overhang for Cpf1).

We co-administered newborn C57BL6/J mice with AAV vectors expressing SaCas9, LbCpf1, or AsCpf1 nucleases at a dose of $3 \times 10^{11} \mathrm{GC} /$ mouse together with vectors expressing the corresponding guide RNAs at a dose of $2 \times 10^{12} \mathrm{GC} /$ mouse (sgRNA, Table 1 ). We sacrificed mice on day 21 post-vector administration, harvested the liver, and extracted the DNA for ITR-Seq analysis in order to evaluate the frequency and location of nuclease-mediated DNA cleavage sites. We coadministered additional groups of newborn mice with vectors expressing SaCas9 or LbCpf1 as above at a dose of $10^{11}$ or $3 \times 10^{11} \mathrm{GC} / \mathrm{mouse}$. The second vector expressed sgRNA and the human coagulation factor IX (hFIX) transgene (instead of the donor DNA sequence used in the first experiment) at a dose of $10^{12} \mathrm{GC} /$ mouse. Our goal was to evaluate the effect of vector dose on ITR integration. We sacrificed these mice on day 70 post-vector administration and subjected DNA samples from the liver to ITR-Seq analysis (Table 1).

We administered AAV8 expressing the indicated nucleases to mice at the indicated doses. This table shows the targeted gene and genomic position, on-target indel percentage, and the number of ITR-Seq-identified offtarget sites.

Aside from one animal treated with AsCpf1-sgRNA2, the on-target locus (mASS1) was the target with the highest number of reads in ITR-Seq analysis. In addition, all of the evaluated sgRNA-directed nucleases exhibited high specificity for the targeted locus with an on-target

Table 1 Using ITR-Seq for on- and off-target evaluation of sgRNA-dependent nucleases

\begin{tabular}{|c|c|c|c|c|c|c|c|c|}
\hline \multirow[t]{2}{*}{$\begin{array}{l}\text { Vector } 1 \\
\text { (GC/kg dose) }\end{array}$} & \multirow[t]{2}{*}{$\begin{array}{l}\text { Vector } 2 \\
\text { (GC/kg dose) }\end{array}$} & \multirow[t]{2}{*}{$\begin{array}{l}\text { Time } \\
\text { point(days) }\end{array}$} & \multicolumn{2}{|c|}{$\begin{array}{l}\text { Indel\% } \\
\text { (on-target) }\end{array}$} & \multicolumn{2}{|c|}{ Off-Target Sites } & \multirow[t]{2}{*}{ Target locus } & \multirow[t]{2}{*}{ Target Sequence } \\
\hline & & & $\begin{array}{l}\text { Mouse } \\
\text { A }\end{array}$ & $\begin{array}{l}\text { Mouse } \\
\text { B }\end{array}$ & $\begin{array}{l}\text { Mouse } \\
\text { A }\end{array}$ & $\begin{array}{l}\text { Mouse } \\
\text { B }\end{array}$ & & \\
\hline $\begin{array}{l}\text { AAV8-SaCas9 } \\
\left(3 \times 10^{11}\right)\end{array}$ & $\begin{array}{l}\text { AAV8-sgRNA1 } \\
\left(2 \times 10^{12}\right)\end{array}$ & 21 & 28.05 & 32.59 & 3 & 4 & ASS1 (chr2:31518639-31518658) & ACAGGACTCCCAGAGTTAGA \\
\hline $\begin{array}{l}\text { AAV8-LbCpf1 } \\
\left(3 \times 10^{11}\right)\end{array}$ & $\begin{array}{l}\text { AAV8-sgRNA1 } \\
\left(2 \times 10^{12}\right)\end{array}$ & 21 & 14.35 & 23.87 & 1 & 2 & ASS1 (chr2:31518480-31518502) & CAAATGGCAGGAAGAATTCACGG \\
\hline $\begin{array}{l}\text { AAV8-LbCpf1 } \\
\left(3 \times 10^{11}\right)\end{array}$ & $\begin{array}{l}\text { AAV8-sgRNA2 } \\
\left(2 \times 10^{12}\right)\end{array}$ & 21 & 33.42 & 27.64 & 4 & 3 & ASS1 (chr2:31519012-31519034) & TGGCTGGAAATATTAGGGCAACT \\
\hline $\begin{array}{l}\text { AAV8-AsCpf1 } \\
\left(3 \times 10^{11}\right)\end{array}$ & $\begin{array}{l}\text { AAV8-sgRNA1 } \\
\left(2 \times 10^{12}\right)\end{array}$ & 21 & 1.22 & 0.38 & 3 & 1 & ASS1 (chr2:31518480-31518502) & CAAATGGCAGGAAGAATTCACGG \\
\hline $\begin{array}{l}\text { AAV8-AsCpf1 } \\
\left(3 \times 10^{11}\right)\end{array}$ & $\begin{array}{l}\text { AAV8-sgRNA2 } \\
\left(2 \times 10^{12}\right)\end{array}$ & 21 & 0.28 & 0.12 & 2 & 1 & ASS1 (chr2:31519012-31519034) & TGGCTGGAAATATTAGGGCAACT \\
\hline $\begin{array}{l}\text { AAV8-SaCas9 } \\
\left(3 \times 10^{11}\right)\end{array}$ & $\begin{array}{l}\text { AAV8-sgRNA1 } \\
\left(1 \times 10^{12}\right)\end{array}$ & 70 & 26.94 & 29.11 & 10 & 12 & ASS1 (chr2:31518639-31518658) & ACAGGACTCCCAGAGTTAGA \\
\hline $\begin{array}{l}\text { AAV8-SaCas9 } \\
\left(1 \times 10^{11}\right)\end{array}$ & $\begin{array}{l}\text { AAV8-sgRNA1 } \\
\left(1 \times 10^{12}\right)\end{array}$ & 70 & 21.39 & 25.98 & 7 & 9 & ASS1 (chr2:31518639-31518658) & ACAGGACTCCCAGAGTTAGA \\
\hline $\begin{array}{l}\text { AAV8-LbCpf1 } \\
\left(1 \times 10^{11}\right)\end{array}$ & $\begin{array}{l}\text { AAV8-sgRNA2 } \\
\left(1 \times 10^{12}\right)\end{array}$ & 70 & 4.91 & 4.90 & 5 & 6 & ASS1 (chr2:31519012-31519034) & TGGCTGGAAATATTAGGGCAACT \\
\hline $\begin{array}{l}\text { AAV8-LbCpf1 } \\
\left(3 \times 10^{11}\right)\end{array}$ & $\begin{array}{l}\text { AAV8-sgRNA2 } \\
\left(1 \times 10^{12}\right)\end{array}$ & 70 & 7.69 & 8.48 & 10 & 4 & ASS1 (chr2:31519012-31519034) & TGGCTGGAAATATTAGGGCAACT \\
\hline
\end{tabular}


indel percentage of up to 33\% (Table 1). Importantly, mice treated with AAV8-SaCas9 exhibited the highest frequency of on-target ITR integration events across all treated samples (Table 1). Despite showing comparable on-target editing, we observed lower on-target ITR integration events in livers treated with AAV8-LbCpf1 versus AAV8-SaCas9 (S3 Table). AAV8-AsCpf1 had very low editing efficiency at the on-target locus with a maximum indel percentage of $1.22 \%$, as assessed by targeted amplicon sequencing (Table 1).

All of the ITR-Seq-identified off-target sites for the CRISPR nucleases resided within annotated mouse genes, with the most common site being the on-target locus (S3 Table). We identified some low-frequency offtarget sites for the tested sgRNAs, most of which had low homology to the target sequence. One off-target site that we identified for the AAV8-SaCas9 sgRNA was located within the locus of a known oncogene, NOTCH2 (S3 Table). Importantly, we found this off-target site in mice administered with AAV8-SaCas9 at a dose of $3 \times$ $10^{11} \mathrm{GC} /$ mouse and AAV-sgRNA1 at a dose of $2 \times 10^{12}$ $\mathrm{GC} /$ mouse. However, this site was absent following administration of $10^{12} \mathrm{GC} /$ mouse of AAV-sgRNA, a twofold lower dose. Thus, only a higher dose of AAV8sgRNA1 led to editing at this low abundance off-target site (Table 1 and S3 Table).
Analyzing nuclease-independent events in mice and nonhuman primates

Among mice injected with AAV8 expressing a CRISPRrelated nuclease and an sgRNA, ITR integration occurred in the loci targeted by the sgRNA. Interestingly, ITR integration also occurred in a seemingly sgRNA-independent fashion, as we observed AAV ITR integration in control samples where there was no functional sgRNA (Table 2) [33, 34]. To evaluate the frequency and scope of nuclease-independent ITR integration in non-human primates, we evaluated the liver DNA of rhesus macaques administered with AAV-eGFP at a dose of $3 \times 10^{12} \mathrm{GC} / \mathrm{kg}$ (Table 2). We detected nucleaseindependent integration events, although not in identified genes, as positive ITR insertions. Importantly, our method appears to exclusively identify integrated ITR sequences as a result of in vivo events. We came to this conclusion based on our finding that we did not identify off-target sites in DNA isolated from PBMCs of untreated rhesus macaques spiked with AAV.eGFP DNA (data not shown).

This table shows a rank-ordered list of identified nuclease off-target sites and the corresponding genome location. For AAV8.EGFP treatments, we injected macaques with AAV at day 7,35 , or $45(\mathrm{~d} 7, \mathrm{~d} 35$, or $\mathrm{d} 45$, respectively), and then euthanized them at either one week or one-month post vector administration ( $1 \mathrm{w}$ or $1 \mathrm{~m})$.

Table 2 Annotation of nuclease-independent, ITR-Seq-identified off-target sites to genomic location

\begin{tabular}{|c|c|c|c|c|}
\hline Sample & & Genomic location & ITR-Seq reads & Gene Symbol \\
\hline AAV8-SaCas9 + AAV8-sgRNA-ctrl & Mouse A & 2:98666941-98666946 & 5 & Gm10800 \\
\hline AAV8-SaCas9 + AAV8-sgRNA-ctrl & Mouse B & 5:90465978-90465995 & 8 & Alb \\
\hline AAV8-LbCpf1 + AAV8-sgRNA-ctrl & Mouse A & - & - & - \\
\hline \multirow[t]{3}{*}{ AAV8-LbCpf1 + AAV8-sgRNA-ctrl } & Mouse B & 2:98667196-98667216 & 29 & Gm10800 \\
\hline & & 2:31519035-31519051 & 22 & Ass1 \\
\hline & & 5:90474802-90474819 & 3 & Alb \\
\hline AAV8-AsCpf1 + AAV8-sgRNA-ctrl & Mouse A & - & - & - \\
\hline \multirow[t]{3}{*}{ AAV8-AsCpf1 + AAV8-sgRNA-ctrl } & Mouse B & 2:31518461-31518481 & 106 & Ass1 \\
\hline & & 5:90472660-90472675 & 7 & Alb \\
\hline & & 9:103219199-103219216 & 3 & Trf \\
\hline AAV8-SaCas9 + AAV8-sgRNA-ctrl & Mouse A & - & - & - \\
\hline \multirow[t]{6}{*}{ AAV8-SaCas9 + AAV8-sgRNA-ctrl } & Mouse B & 2:98666940-98666966 & 67 & Gm10800 \\
\hline & & 5:90476386-90476411 & 12 & Alb \\
\hline & & 5:90472060-90472079 & 11 & Alb \\
\hline & & 9:21837574-21837595 & 10 & Dock6 \\
\hline & & 9:692444457-69244483 & 5 & Rora \\
\hline & & 5:90505454-90505480 & 4 & Alb \\
\hline AAV8-LbCpf1 + AAV8-sgRNA-ctrl & Mouse A & - & - & - \\
\hline AAV8-LbCpf1 + AAV8-sgRNA-ctrl & Mouse B & - & - & - \\
\hline AAV8.EGFP (rhesus macaque) & $d 7-1 w$ & 6:157029767-157029776 & 12 & - \\
\hline AAV8.EGFP (rhesus macaque) & $d 35-1 w$ & 2:7161434-7161476 & 104 & \\
\hline AAV8.EGFP (rhesus macaque) & $\mathrm{d} 45-1 \mathrm{~m}$ & - & - & - \\
\hline
\end{tabular}




\section{Discussion}

Developing an NGS-based assay that can identify and rank nuclease-induced DSBs after in vivo gene editing would significantly advance our ability to evaluate the safety and efficacy of genome editing therapies for translation to human clinical trials. Here, we show that our novel ITR-Seq assay can assess the specificity of any AAV-expressed nuclease in vivo. This method is more sensitive in detecting ITR integration than other NGSbased methods such as the combined approach of GUIDE-Seq and subsequent amplicon sequencing.

In contrast to GUIDE-Seq, ITR-Seq is not a tool for predicting in vivo off-target sites. Rather, ITR-Seq identifies novel sites in the genome where on- and off-target nuclease activity occurred. Indeed, this method identifies AAV ITR integration sites directly from the DNA samples of animals treated with nuclease-expressing AAV. Identified off-target sites can be further analyzed using amplicon sequencing to 1) accurately determine the percent of editing and ITR integration; and 2) obtain a detailed panorama of the nuclease activity in clinically relevant doses and animal models.

Analysis of non-human primate samples by ITR-Seq showed a clear decrease in the number of off-target sites, as well as the total number of ITR-Seq reads for the offtarget sequences from d17 to d128 (Fig. 2a). Considering that we observed a relationship between the total AAV dose and the total number of off-target sites (Fig. 2a), we hypothesize that high intracellular expression of the nuclease, as a consequence of the high AAV dose, results in a high number of off-target sites. If these cells are later removed either by the immune system or due to toxicity related to the off-target activity, then a decrease in the total number of off-target sites is expected. More experiments are needed to correctly identify the cause of this off-target reduction over time.

In our studies in mice, the most common nucleaseindependent ITR integration events occurred in the Gm10800 and albumin genes. ITR integration in the albumin gene concurs with previous reports that show that the albumin gene is quite susceptible to AAV integration [33]. Furthermore, AAV integration has been reported for genes that are transcriptionally active in the liver [34]. Our findings suggest that the rate of AAV ITR integration can be influenced by 1) the homology between the target sequence; or 2) the blunt or overhang nature of the DNA ends as a result of the nuclease cuts. Detailed studies in the future will be needed to fully elucidate the dynamics of ITR integration.

Alternative methods such as BLISS [17], BLESS [35], and End-Seq [18] can identify sites of DSBs by capturing the DSB ends created by the activity of the nuclease. These methods can accurately identify nuclease-induced DSBs in vivo. However, these methods have the limitation that they can only capture DSBs present at a single time point. This limitation can be partially overcome by analyzing DSBs at multiple time points. Non-restrictive linear-amplification mediated PCR (nrLAM-PCR) coupled with NGS [36], a method similar to ITR-Seq, can detect AAV-ITR integration sites. Researchers have used this method to identify the integration sites of the AAV1-LPLS447X vector, which was developed for treating lipoprotein lipase deficiency in mouse and human DNA samples [29]. Although in theory nrLAM-PCR can detect the off-target activity of nucleases, researchers have not yet directly compared ITR-Seq and nrLAMPCR to understand the advantages and limitations of these two techniques.

\section{Conclusions}

In conclusion, given that the only requirement of ITRSeq is using an AAV as a delivery vector, this method can be used to measure the specificity of the nucleases in virtually any organism with an annotated reference genome. Researchers could use ITR-Seq as a companion diagnostic in pre-clinical and clinical studies to evaluate nuclease target sites in longitudinal animal studies that have varying dosages and/or administration routes. This technique can yield invaluable insights into the safety and efficacy of gene editing therapies and ultimately better inform the design of future gene editing therapies. This same approach can evaluate AAV integration events in traditional AAV gene therapy studies. Although the risks of insertional mutagenesis from AAV gene therapy is considered low enough $[19,37]$ to justify its use for treating rare disabling and lethal diseases, these potential risks may be more relevant as the field evolves to treating less severe acquired diseases.

\section{Methods}

\section{Animal studies}

All animal procedures were performed in accordance with protocols approved by the Institutional Animal Care and Use Committee of the University of Pennsylvania. Mice were obtained from The Jackson Laboratory (Bar Harbor, ME).

\section{Rhesus macaque studies}

DNA samples from previously published studies [26] were used for ITR-Seq analysis. Briefly, AAV8 vectors driving the expression of the meganucleases M1PCSK9 (AAV8.T BG.M1PCSK9.WPRE) or M2PCSK9 (AAV8.TBG.M2PCS K9.WPRE) were administered via a peripheral vein to rhesus macaques $(n=4$ for M1PCSK9- and $n=2$ for M2PCSK9-treated animals). Liver biopsies were performed at 17 and 129 days (for AAV8-M1PCSK9) or 18 and 128 days (for AAV8-M2PCSK9) post vector administration [26]. As an untreated control, we used DNA extracted from 
PBMC samples collected prior to vector administration [26].

To measure nuclease-independent AAV integration events, we analyzed liver DNA samples from a previous published study [38]. Briefly, one-week or one-monthold male rhesus macaques were administered with AAV8.TBG.EGFP at a dose of $3 \times 10^{12} \mathrm{GC} / \mathrm{kg}$. Animals were euthanized post-vector administration, and livers were collected.

\section{Mouse studies}

Newborn ( $0-2$ days of age, $n=2$ per group) C57BL/6 J mice were co-administered by temporal vein injection AAV expressing either SaCas9 (AAV8.TBG.hSaCas9.bGH), LbCpf1 (AAV8.ABP2.TBG-S1.hLbCpf1.bGH), or AsCpf1 (AAV8.A BPS2.TBG-S1.hAsCpf1.PA75) at a dose of $3 \times 10^{11} \mathrm{GC} /$ mouse, as well as vectors expressing specific sgRNA (AAV8.U6.sgRNA.mASS1.donor(mASS1)) or untargeted sgRNA as controls (AAV8.U6.sgRNA-ctrl.mASS1.donor(mASS1)) at a dose of $2 \times 10^{12} \mathrm{GC} /$ mouse. At 21 days postvector administration, mice were euthanized by carbon dioxide asphyxiation, death was confirmed by cervical dislocation, and livers were collected.

Additional newborn mice ( $n=2$ per group) were coadministered with vector expressing SaCas9 or LbCpf1 as described above at a dose of $10^{11}$ or $3 \times 10^{11} \mathrm{GC} /$ mouse, with the second vector expressing ASS1-specificsgRNA and the hFIX transgene (AAV8.U6.sgRNA.mASS1.TBG.hFIX) at a dose of $10^{12} \mathrm{GC} /$ mouse. Livers were collected at 70 days post-vector administration.

\section{ITR-Seq}

The ITR-Seq protocol is a modified version of an anchored PCR reaction [12, 32], in which a single primer is designed to anneal to and amplify outward from the ITR sequence (Fig. 1c). Following ITR integration in the DNA, the primer may be used to amplify the junction of the host genome and the inserted vector ITR sequence (Fig. 1b, c). In order to adequately denature the ordered secondary structure of the integrated ITR, we used a high annealing temperature of $69^{\circ} \mathrm{C}$ and designed longer adapter-specific primers (S4 Table).

Amplicons were generated from purified genomic DNA isolated from liver tissue samples. DNA was sheared to an average size of $500 \mathrm{bp}$ using an ME220 focusedultrasonicator (Covaris, Woburn, MA), purified using AMPure beads (Beckman Coulter, Indianapolis, IN) at a $0.8 \mathrm{x}$ ratio, and eluted in $15 \mu \mathrm{l}$ of elution buffer (Qiagen, Hilden, Germany). End repair was subsequently performed in a total volume of $22.5 \mu \mathrm{l}$ containing $1 \mu \mathrm{l}$ of $5 \mathrm{mM}$ dNTP mix (Thermo Fisher Scientific, Waltham, MA), $2.5 \mu \mathrm{l}$ of 10x SLOW ligation buffer (Enzymatics, Beverly, MA), $2 \mu \mathrm{l}$ of End-Repair Mix (Low Concentration; Enzymatics, Beverly, MA), $2 \mu \mathrm{l}$ of $10 \mathrm{x}$ buffer for Taq Polymerase $\left(\mathrm{MgCl}_{2}\right.$ - free; Invitrogen, Carlsbad, CA), $0.5 \mu \mathrm{l}$ of non-hot start Taq polymerase (New England BioLabs, Ipswich, MA), $0.5 \mu \mathrm{l}$ of nuclease-free water (Life Technologies, Waltham, MA), and $14 \mu \mathrm{l}$ of $400 \mathrm{ng}$ sheared genomic DNA. The mix was incubated at $12{ }^{\circ} \mathrm{C}$ for $15 \mathrm{~min}, 37^{\circ} \mathrm{C}$ for $15 \mathrm{~min}, 72^{\circ} \mathrm{C}$ for $15 \mathrm{~min}$, and then held at $4{ }^{\circ} \mathrm{C}$. Unique Y-adapters, with molecular index tags annealed to MiSeq Common Adapters (Illumina, San Diego, CA), were ligated to the end-repaired DNA in the following mix: $1 \mu \mathrm{l}$ of $10 \mu \mathrm{M}$ annealed A01A16 Y-adapter, $2 \mu \mathrm{l}$ of T4 DNA ligase (Enzymatics, Beverly, MA), and $22.5 \mu \mathrm{l}$ of the previous end-repaired DNA. The ligation program was $16^{\circ} \mathrm{C}$ for $30 \mathrm{~min}, 22^{\circ} \mathrm{C}$ for $30 \mathrm{~min}$, and then held at $4{ }^{\circ} \mathrm{C}$. DNA was then purified by AMPure beads (Beckman Coulter, Indianapolis, IN) at a $0.7 x$ ratio. End-repaired Y-adapter-ligated DNA fragments were amplified by PCR using an ITR-specific primer and an adapterspecific primer (A01-A16_P5_FWD primer) in the following mix (amounts per sample): $11.9 \mu \mathrm{l}$ of nuclease-free water, $3 \mu \mathrm{l}$ of $10 \mathrm{x}$ buffer for Taq Polymerase $\left(\mathrm{MgCl}_{2}\right.$-free, Invitrogen, Carlsbad, CA), $0.6 \mu \mathrm{l}$ of $10 \mathrm{mM}$ dNTP mix (Thermo Fisher Scientific, Waltham, MA), $1.2 \mu \mathrm{l}$ of $50 \mathrm{mM}$ $\mathrm{MgCl}_{2}$ (Invitrogen, Carlsbad, CA), $0.3 \mu \mathrm{l}$ of $5 \mathrm{U} / \mu \mathrm{l}$ Platinum Taq polymerase (Invitrogen, Carlsbad, CA), $1 \mu \mathrm{l}$ of $10 \mu \mathrm{M}$ GSP_ITR3.AAV2 primer, $1.5 \mu \mathrm{l}$ of $0.5 \mathrm{M}$ TMAC (SigmaAldrich, St. Louis, MO), $0.5 \mu \mathrm{l}$ of $10 \mu \mathrm{M}$ A01-A16_P5_ FWD primer with the primer number matching the adapter number (e.g., A01_P5_FWD primer to be used with A01 Yadapter), and $10 \mu$ lof previously purified DNA. The PCR program was 1 cycle of $95^{\circ} \mathrm{C}$ for $5 \mathrm{~min} 30$ cycles of $95^{\circ} \mathrm{C}$ for $30 \mathrm{~s}, 69^{\circ} \mathrm{C}$ for $1 \mathrm{~min}$, and $72^{\circ} \mathrm{C}$ for $30 \mathrm{~s} ; 1$ cycle at $72{ }^{\circ} \mathrm{C}$ for $5 \mathrm{~min}$; and $4{ }^{\circ} \mathrm{C}$ hold. PCR products were purified using 0.7x AMPure beads (Beckman Coulter, Indianapolis, IN) and resuspended in $15 \mu \mathrm{l}$ of elution buffer (Qiagen, Hilden, Germany).

NGS libraries were prepared by PCR in the following mix (amounts per sample): $5.4 \mu \mathrm{l}$ of nuclease-free water (Life Technologies, Waltham, MA), $3 \mu \mathrm{l}$ of 10x buffer for Taq Polymerase $\left(\mathrm{MgCl}_{2}\right.$-free; Invitrogen, Carlsbad, CA), $0.6 \mu \mathrm{l}$ of $10 \mathrm{mM} \mathrm{dNTP}$ mix (Thermo Fisher Scientific, Waltham, MA), $1.2 \mu \mathrm{l}$ of $50 \mathrm{mM} \mathrm{MgCl}_{2}$ (Invitrogen, Carlsbad, CA), $0.3 \mu \mathrm{l}$ of $5 \mathrm{U} / \mu$ l Platinum Taq polymerase (Invitrogen, Carlsbad, CA), $1 \mu \mathrm{l}$ of $10 \mu \mathrm{M}$ GSP_ITR3 primer, $1.5 \mu \mathrm{l}$ of $0.5 \mathrm{M}$ TMAC (Sigma-Aldrich, St. Louis, $\mathrm{MO}), 0.5 \mu \mathrm{l}$ of $10 \mu \mathrm{M}$ A01-A16_P5_FWD primer with the primer number matching the adapter number, $1.5 \mu \mathrm{l}$ of $10 \mu \mathrm{M} \mathrm{p} 701-16$ primers, and $15 \mu \mathrm{l}$ of previously purified DNA (including the AMPure beads used in the previous PCR purification step). The PCR program was 1 cycle of $95^{\circ} \mathrm{C}$ for $5 \mathrm{~min} ; 10$ cycles of $95^{\circ} \mathrm{C}$ for $30 \mathrm{~s}, 75^{\circ} \mathrm{C}$ for $2 \mathrm{~min}\left(-1{ }^{\circ} \mathrm{C} /\right.$ cycle $)$, and $72^{\circ} \mathrm{C}$ for $30 \mathrm{~s} ; 15$ cycles of $95^{\circ} \mathrm{C}$ for $30 \mathrm{~s}, 69^{\circ} \mathrm{C}$ for $1 \mathrm{~min}$, and $72^{\circ} \mathrm{C}$ for $30 \mathrm{~s} ; 1$ cycle at $72{ }^{\circ} \mathrm{C}$ for $5 \mathrm{~min}$; and $4{ }^{\circ} \mathrm{C}$ hold. PCR products were purified using 0.7x AMPure beads (Beckman Coulter, Indianapolis, IN), and resuspended in $25 \mu \mathrm{l}$ of elution 
buffer. Dual-indexed sequencing libraries were sequenced on an Illumina MiSeq cartridge (MiSeq ${ }^{\circ}$ v2 RGT Kit 300 cyc PE-Bx 1 of 2; San Diego, CA), generating $2 \times 150$ bp paired-end reads.

Sample demultiplexing and unique molecular identifier (UMI) tagging was performed on raw fastq files using Je [39], allowing for up to one mismatch on either index. Read pairs in which read 2 begins with the designed primer sequence, plus an additional flanking $20 \mathrm{bp}$ of the AAV2 ITR sequence (Fig. 1), were identified using FASTX barcode slitter (http://hannonlab.cshl.edu/fastx_toolkit/, allowing for up to five mismatches), fastq-pair (https://github.com/linsalrob/EdwardsLab/), and FASTP [40]. Selected read pairs were mapped to the reference genome for each sample (MM10 for mouse and RheMac8 for rhesus macaque samples) using NovoAlign (Novocraft, Selangor, Malaysia). UMI read consolidation was subsequently performed using Je [39], resulting in a UMI-consolidated BAM file. Chimeric reads spanning the ITR-genomic DNA insertion site were identified by determining split-read junctions for each read using SE-MEI (https:/github.com/dpryan79/SE-MEI). Softclipped portions of reads were then mapped to the AAV2 reference genome using NovoAlign (Novocraft, Selangor, Malaysia). Only those originally mapped reads that were found to contain a soft-clipped read portion mapping to the AAV2 ITR with a mapping quality value greater than or equal to 30 were used to identify ITR integration sites by merging ITR integration sites found within a 50 bp window into a single ITR integration site using BEDtools [41]. Only those sites with an ITR integrated in both the forward and negative strand orientations were considered to be an identified off-target site. The genomic DNA sequence underlying each identified ITR integration site was aligned pairwise with the on-target DNA sequence motif from both the negative and positive strand orientations using the EMBOSS program [42] for semi-global alignment; this allowed us to assess sequence homology and predict the nuclease target sequence for each ITR integration site.

\section{Supplementary information}

Supplementary information accompanies this paper at https://doi.org/10. 1186/s 12864-020-6655-4.

Additional file1 Supporting Dataset 1. Supporting Dataset 2. S1 Table Validating ITR-Seq-identified off-target events. S2 Table. ITR-Seq rank of GUIDE-Seq-identified off-target events. S3 Table. Annotation of ITR-Seqidentified on- and off-target events. S4 Table. List of primer sequences used in this study.

Additional file 2 Supplemental Figure 1. Frequency of AAV integration in the on- and off-target sites. The number of ITR-Seq reads for the on and off-target sites are shown as a percentage of the total number of ITR-Seq reads before the filtering step (see Methods). Analysis was performed on the ITR-Seq results for liver biopsies at d17 and d128 from non-human primates treated with the indicated nuclease and AAV dose.
Additional file $\mathbf{3}$ Supplemental Figure $\mathbf{2}$. Distribution of mismatches between the target sequence and identified off-target sequences. Offtargets sequences were extracted from the ITR-Seq results for AAVM1PCSK9 (at a dose of $3 \times 10^{13}$ or $6 \times 10^{12} \mathrm{GC} / \mathrm{Kg}$, panels a and $\mathrm{b}$ ) and AAV-M2PCSK9 $\left(6 \times 10^{12} \mathrm{GC} / \mathrm{kg}\right.$ dose, panels $\mathrm{c}$ and $\left.\mathrm{d}\right)$ groups at $\mathrm{d} 17$. Thirty-one top-ranked (according to the number of ITR-Seq reads) offtarget sequences, with a length of $22 \mathrm{bp}$ and with no more than 10 mismatches, were retained for analysis. Location of the off-target sites are shown on the left and mismatches between the off- and on-target sequences are highlighted. The data to generate the WebLogo [43] shown on top were the selected off-target sequences for each group multiplied by the reported number of ITR-Seq reads (Dataset S1).

\section{Abbreviations}

AAV: Adeno-associated viral; BLESS: Breaks labeling, enrichment on streptavidin and sequencing; BLISS: Breaks labeling in situ and sequencing; CRISPR: Clusters of regularly interspersed short palindromic repeats; DNA: Deoxyribonucleic acid; dNTP: Deoxyribonucleotide triphosphate; DSB: Double-stranded breaks; EMBOSS: European Molecular Biology Open Software Suite; GC: Genome copies; hFIX: Human coagulation factor IX; ITR: Inverted terminal repeat; ITR-Seq: ITR sequencing; NGS: Next-generation sequencing; nrLAM-PCR: Non-restrictive linear-amplification mediated PCR; PBMC: Peripheral blood mononuclear cell; PCR: Polymerase chain reaction; PCSK9: Proprotein convertase subtilisin/kexin type 9; RBE: Rep-binding element; RNA: Ribonucleic acid; sgRNA: Single-guide RNA;

TALENS: Transcription activator-like effector nucleases;

TMAC: Tetramethylammonium chloride solution; TRS: Terminal resolution site; TSS: Transcription start sites; TTS: Transcription termination sites; UMI: Unique molecular identifier

\section{Acknowledgements}

We thank the Penn Vector Core, the Program in Comparative Medicine, and the Nucleic Acid Technologies Core at the University of Pennsylvania.

\section{Authors' contributions}

C.B. and J.M.W. conceived and designed the experiments. C.B. performed the experiments. C.B., P.C., J.A.G., and J.M.W. analyzed the data. C.B., P.C., and L.W. contributed reagents and analysis tools. C.B., P.C., J.A.G., and J.M.W. wrote the manuscript together. All authors read and approved the manuscript.

\section{Funding}

This research was supported by grants awarded to J.M.W. from Precision Biosciences and The University of Pennsylvania, Perelman School of Medicine. These funding bodies played no role in any of the following: study design, data collection, analysis, and interpretation; manuscript writing.

\section{Availability of data and materials}

Datasets S1 and S2 are available at BioProject (accession number PRJNA609560).

\section{Ethics approval and consent to participate}

All animal procedures were performed in accordance with protocols approved by the Institutional Animal Care and Use Committee of the University of Pennsylvania. No additional studies in rhesus macaques were performed, only DNA samples from previously published studies [26] [36] were used here. All animal procedures were performed in accordance with protocols approved by the Institutional Animal Care and Use Committee (IACUC) of the University of Pennsylvania. Mouse studies were performed under IACUC protocol 803270 and mice were obtained from The Jackson Laboratory (Bar Harbor, ME). "Consent to participate" is not applicable as this study did not involve human participants.

\section{Consent for publication}

Not applicable as this study did not involve human participants.

\section{Competing interests}

J.M. Wilson is a paid advisor to and holds equity in Scout Bio and Passage Bio; he holds.

equity in Surmount Bio; he also has a sponsored research agreement with Ultragenyx, 
Biogen, Janssen, Precision Biosciences, Moderna Inc., Scout Bio, Passage. Bio, Amicus Therapeutics, and Surmount Bio which are licensees of Penn technology.

JMW is an inventor on patents that have been licensed to various biopharmaceutical.

companies and for which he may receive payments.

\section{Received: 17 December 2019 Accepted: 5 March 2020}

\section{Published online: 17 March 2020}

\section{References}

1. Kosicki M, Tomberg K, Bradley A. Repair of double-strand breaks induced by CRISPR-Cas9 leads to large deletions and complex rearrangements. Nat Biotechnol. 2018;36(8):765-71.

2. Sander JD, Joung JK. CRISPR-Cas systems for editing, regulating and targeting genomes. Nat Biotechnol. 2014;32(4):347-55.

3. Waryah CB, Moses C, Arooj M, Blancafort P. Zinc fingers, TALEs, and CRISPR systems: a comparison of tools for Epigenome editing. Methods Mol Biol. 1767;2018:19-63.

4. Arnould S, Delenda C, Grizot S, Desseaux C, Paques F, Silva GH, et al. The ICrel meganuclease and its engineered derivatives: applications from cell modification to gene therapy. Protein Eng Des Sel. 2011;24(1-2):27-31.

5. Silva G, Poirot L, Galetto R, Smith J, Montoya G, Duchateau P, et al. Meganucleases and other tools for targeted genome engineering: perspectives and challenges for gene therapy. Curr Gene Ther. 2011;11(1):11-27.

6. Martin F, Sanchez-Hernandez S, Gutierrez-Guerrero A, Pinedo-Gomez J, Benabdellah K. Biased and Unbiased Methods for the Detection of OffTarget Cleavage by CRISPR/Cas9: An Overview. Int J Mol Sci. 2016;17(9):1507.

7. Lazzarotto CR, Nguyen NT, Tang X, Malagon-Lopez J, Guo JA, Aryee MJ, et al. Defining CRISPR-Cas9 genome-wide nuclease activities with CIRCLEseq. Nat Protoc. 2018;13(11):2615-42.

8. Yee JK. Off-target effects of engineered nucleases. FEBS J. 2016;283(17):3239-48.

9. Cameron P, Fuller CK, Donohoue PD, Jones BN, Thompson MS, Carter MM, et al. Mapping the genomic landscape of CRISPR-Cas9 cleavage. Nat Methods. 2017;14(6):600-6.

10. Kim D, Bae S, Park J, Kim E, Kim S, Yu HR, et al. Digenome-seq: genomewide profiling of CRISPR-Cas9 off-target effects in human cells. Nat Methods. 2015;12(3):237-43 1 p following 43.

11. Tsai SQ, Nguyen NT, Malagon-Lopez J, Topkar W, Aryee MJ, Joung JK. CIRCLE-seq: a highly sensitive in vitro screen for genome-wide CRISPR-Cas9 nuclease off-targets. Nat Methods. 2017;14(6):607-14.

12. Tsai SQ, Zheng Z, Nguyen NT, Liebers M, Topkar W, Thapar V, et al. GUIDEseq enables genome-wide profiling of off-target cleavage by CRISPR-Cas nucleases. Nat Biotechnol. 2015:33(2):187-97.

13. Gabriel R, Lombardo A, Arens A, Miller JC, Genovese P, Kaeppel C, et al. An unbiased genome-wide analysis of zinc-finger nuclease specificity. Nat Biotechnol. 2011;29(9):816-23.

14. Wang X, Wang Y, Wu X, Wang J, Wang Y, Qiu Z, et al. Unbiased detection of off-target cleavage by CRISPR-Cas9 and TALENs using integrase-defective lentiviral vectors. Nat Biotechnol. 2015;33(2):175-8.

15. Hanlon KS, Kleinstiver BP, Garcia SP, Zaborowski MP, Volak A, Spirig SE, et al. High levels of AAV vector integration into CRISPR-induced DNA breaks. Nat Commun. 2019;10(1):4439. https://doi.org/10.1038/s41467-019-12449-2.

16. Verkuijl SA, Rots MG. The influence of eukaryotic chromatin state on CRISPRCas9 editing efficiencies. Curr Opin Biotechnol. 2019;55:68-73. https://doi. org/10.1016/j.copbio.2018.07.005

17. Yan WX, Mirzazadeh $\mathrm{R}$, Garnerone S, Scott D, Schneider MW, Kallas T, et al. BLISS is a versatile and quantitative method for genome-wide profiling of DNA double-strand breaks. Nat Commun. 2017;8:15058.

18. Canela A, Sridharan S, Sciascia N, Tubbs A, Meltzer P, Sleckman BP, et al. DNA breaks and end resection measured genome-wide by end sequencing. Mol Cell. 2016;63(5):898-911.

19. Chandler RJ, Sands MS, Venditti CP. Recombinant Adeno-associated viral integration and Genotoxicity: insights from animal models. Hum Gene Ther. 2017;28(4):314-22.

20. Janovitz T, Oliveira T, Sadelain M, Falck-Pedersen E. Highly divergent integration profile of adeno-associated virus serotype 5 revealed by highthroughput sequencing. J Virol. 2014;88(5):2481-8.

21. Kotin RM, Siniscalco M, Samulski RJ, Zhu XD, Hunter L, Laughlin CA, et al. Site-specific integration by adeno-associated virus. Proc Natl Acad Sci U S A. 1990;87(6):2211-5
22. Miller DG, Trobridge GD, Petek LM, Jacobs MA, Kaul R, Russell DW. Largescale analysis of adeno-associated virus vector integration sites in normal human cells. J Virol. 2005;79(17):11434-42.

23. Nakai $H$, Wu X, Fuess S, Storm TA, Munroe D, Montini E, et al. Large-scale molecular characterization of adeno-associated virus vector integration in mouse liver. J Virol. 2005;79(6):3606-14.

24. Nault JC, Datta S, Imbeaud S, Franconi A, Mallet M, Couchy G, et al. Recurrent AAV2-related insertional mutagenesis in human hepatocellular carcinomas. Nat Genet. 2015;47(10):1187-93.

25. Samulski RJ, Zhu X, Xiao X, Brook JD, Housman DE, Epstein N, et al. Targeted integration of adeno-associated virus (AAV) into human chromosome 19. EMBO J. 1991;10(12):3941-50.

26. Wang L, Smith J, Breton C, Clark P, Zhang J, Ying L, et al. Meganuclease targeting of PCSK9 in macaque liver leads to stable reduction in serum cholesterol. Nat Biotechnol. 2018;36(8):717-25.

27. Miller DG, Petek LM, Russell DW. Adeno-associated virus vectors integrate at chromosome breakage sites. Nat Genet. 2004;36(7):767-73.

28. Donsante A, Miller DG, Li Y, Vogler C, Brunt EM, Russell DW, et al. AAV vector integration sites in mouse hepatocellular carcinoma. Science. 2007;317(5837):477.

29. Kaeppel C, Beattie SG, Fronza R, van Logtenstein R, Salmon F, Schmidt S, et al. A largely random AAV integration profile after LPLD gene therapy. Nat Med. 2013:19(7):889-91.

30. Kotin RM, Menninger JC, Ward DC, Berns Kl. Mapping and direct visualization of a region-specific viral DNA integration site on chromosome 19q13-qter. Genomics. 1991;10(3):831-4.

31. Rosas LE, Grieves JL, Zaraspe K, La Perle KM, Fu H, McCarty DM. Patterns of SCAAV vector insertion associated with oncogenic events in a mouse model for genotoxicity. Mol Ther. 2012;20(11):2098-110.

32. Zheng Z, Liebers M, Zhelyazkova B, Cao Y, Panditi D, Lynch KD, et al. Anchored multiplex PCR for targeted next-generation sequencing. Nat Med. 2014;20(12):1479-84.

33. Chandler RJ, LaFave MC, Varshney GK, Trivedi NS, Carrillo-Carrasco N, Senac $J \mathrm{~S}$, et al. Vector design influences hepatic genotoxicity after adenoassociated virus gene therapy. J Clin Invest. 2015;125(2):870-80.

34. Nakai H, Montini E, Fuess S, Storm TA, Grompe M, Kay MA. AAV serotype 2 vectors preferentially integrate into active genes in mice. Nat Genet. 2003; 34(3):297-302.

35. Crosetto N, Mitra A, Silva MJ, Bienko M, Dojer N, Wang Q, et al. Nucleotideresolution DNA double-strand break mapping by next-generation sequencing. Nat Methods. 2013;10(4):361-5.

36. Paruzynski A, Arens A, Gabriel R, Bartholomae CC, Scholz S, Wang W, et al. Genome-wide high-throughput integrome analyses by nrLAM-PCR and next-generation sequencing. Nat Protoc. 2010;5(8):1379-95.

37. Mingozzi F, High KA. Therapeutic in vivo gene transfer for genetic disease using AAV: progress and challenges. Nat Rev Genet. 2011;12(5):341-55.

38. Wang L, Bell P, Lin J, Calcedo R, Tarantal AF, Wilson JM. AAV8-mediated hepatic gene transfer in infant rhesus monkeys (Macaca mulatta). Mol Ther. 2011;19(11):2012-20.

39. Girardot C, Scholtalbers J, Sauer S, Su SY, Furlong EE. Je, a versatile suite to handle multiplexed NGS libraries with unique molecular identifiers. BMC Bioinformatics. 2016;17(1):419.

40. Chen S, Zhou Y, Chen Y, Gu J. Fastp: an ultra-fast all-in-one FASTQ preprocessor. Bioinformatics. 2018;34(17):i884-i90.

41. Quinlan AR. BEDTools: The Swiss-Army Tool for Genome Feature Analysis. Curr Protoc Bioinformatics. 2014:47:11 2 1-34.

42. Rice P, Longden I, Bleasby A. EMBOSS: the European molecular biology open software suite. Trends Genet. 2000;16(6):276-7.

43. Crooks GE, Hon G, Chandonia JM, Brenner SE. WebLogo: a sequence logo generator. Genome Res. 2004;14(6):1188-90. https://doi.org/10.1101/gr.849004.

\section{Publisher's Note}

Springer Nature remains neutral with regard to jurisdictional claims in published maps and institutional affiliations. 